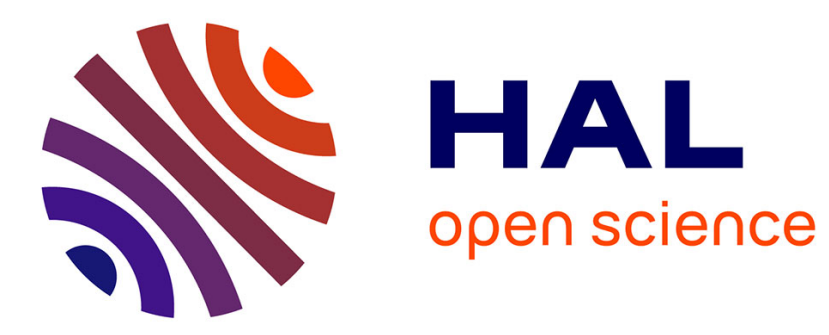

\title{
Oral vaccination against plague using Yersinia pseudotuberculosis
}

\author{
Christian E. Demeure, Anne Derbise, Elisabeth Carniel
}

\section{To cite this version:}

Christian E. Demeure, Anne Derbise, Elisabeth Carniel. Oral vaccination against plague using Yersinia pseudotuberculosis. Chemico-Biological Interactions, 2017, 267, pp.89-95. 10.1016/j.cbi.2016.03.030 . pasteur-02068312

\section{HAL Id: pasteur-02068312 \\ https://hal-pasteur.archives-ouvertes.fr/pasteur-02068312}

Submitted on 14 Mar 2019

HAL is a multi-disciplinary open access archive for the deposit and dissemination of scientific research documents, whether they are published or not. The documents may come from teaching and research institutions in France or abroad, or from public or private research centers.
L'archive ouverte pluridisciplinaire HAL, est destinée au dépôt et à la diffusion de documents scientifiques de niveau recherche, publiés ou non, émanant des établissements d'enseignement et de recherche français ou étrangers, des laboratoires publics ou privés. 


\section{Oral vaccination against plague using Yersinia pseudotuberculosis}

Christian E. Demeure $^{\mathrm{a}^{*}}$, Anne Derbise ${ }^{\mathrm{a}}$ and Elisabeth Carniel $^{\mathrm{a}}$.

a Unité de recherche Yersinia, Institut Pasteur, 28 rue du Dr Roux, 75724 Paris, France.

Christian E. Demeure: cdemeure@pasteur.fr

Anne Derbise: anne.derbise@pasteur.fr

Elisabeth Carniel: elisabeth.carniel@pasteur.fr

Key words: live vaccine; Yersinia pestis; F1 pseudocapsule, bubonic plague; pneumonic plague

Short title: Live oral vaccination against pneumonic and bubonic plague

*: Corresponding author : Dr C.E. Demeure,

Tel: (33-1) 45.68.84.48 Fax: (33-1) 45.68.89.54 


\section{Abstract}

Yersinia pestis, the agent of plague, is among the deadliest bacterial pathogens affecting humans, and is a potential biological weapon. Because antibiotic resistant strains of $Y$. pestis have been observed or could be engineered for evil use, vaccination against plague might become the only means to reduce mortality. Although plague is re-emerging in many countries, a vaccine with worldwide license is currently lacking. The vaccine strategy described here is based on an oral vaccination with an attenuated strain of Yersinia pseudotuberculosis. Indeed, this species is genetically almost identical to Y.pestis, but has a much lower pathogenicity and a higher genomic stability. Gradual modifications of the wild-type Y. pseudotuberculosis strain IP32953 were performed to generate a safe and immunogenic vaccine. Genes coding for three essential virulence factors were deleted from this strain. To increase cross-species immunogenicity, an F1encapsulated Y.pseudotuberculosis strain was then generated. For this, the $Y$. pestis caf operon, which encodes $\mathrm{F} 1$, was inserted first on a plasmid, and subsequently into the chromosome. The successive steps achieved to reach maximal vaccine potential are described, and how each step affected bacterial virulence and the development of a protective immune response is discussed. The final version of the vaccine, named $\mathrm{VTnF} 1$, provides a highly efficient and longlasting protection against both bubonic and pneumonic plague after a single oral vaccine dose. Since a $Y$. pestis strain deprived of $F 1$ exist or could be engineered, we also analyzed the protection conferred by the vaccine against such strain and found that it also confers full protection against the two forms of plague. Thus, the properties of VTnF1 makes it one of the most efficient candidate vaccine for mass vaccination in tropical endemic areas as well as for populations exposed to bioterrorism.

\section{Highlights}

- $\mathrm{VTnF} 1$ is an irreversibly attenuated Yersinia pseudotuberculosis vaccine strain

- VTnF1 produces the Yersinia pestis F1 surface pseudocapsule

- A single oral dose induces a strong and long lasting protective immunity

- VTnF1 protects efficiently against both bubonic \& pneumonic plague

- Vaccination also protects against an F1-mutant of Y. pestis 


\section{Vaccination against plague: the context}

The first widely used plague vaccine was the live attenuated Y. pestis EV76 developed and formerly used in Madagascar. EV76 was obtained by repeated subcultures in vitro of an initially virulent strain that led to the loss of a genetic element named High Pathogenicity Island (HPI), which is important for the systemic dissemination of $Y$. pestis. EV76 induces a strong humoral response and protects 
against bubonic plague and pneumonic plague but immunity was not long-lasting [9]. unstable [10,11], a decline in efficiency of the EV76 vaccine preparations used in different countries was observed because of a genetic drift caused by accumulation of mutations and loss of DNA sequences [9-12]. A derivative of the EV76 strain has been developed in Russia [13]. Showing less secondary effects and renamed NIIEG, it is used in the Russian federation and China.

A plague vaccine composed of formalin-killed whole-cell $Y$. pestis was licensed in the USA and UK during the 20th century. It was discontinued because it was reactogenic in humans and conferred only short-term protection [9], requiring annual booster immunizations [9]. The USP killed vaccine was recently discontinued in the USA. No new killed plague vaccine has replaced that one.

The development of new candidate vaccines has received much efforts during the recent years. Some strategies were based on an attenuated strain of $Y$. pestis [14-20] obtained by deletion of virulence genes [21, 22], or by addition of genes, such the IpxL gene modifying the LPS [23]. Introduction of $Y$. pestis antigens into Salmonella [14] and virus vectors [15, 16], as well as DNA [17-20] were also used to produce plague vaccines. At present, none of these was brought to clinical evaluation phases.

The US and UK governments developed molecular plague vaccines, called F1-V and RypVax® respectively, which are patented but not yet licensed. Both are composed of recombinant $Y$. pestis $\mathrm{F} 1$ and $\mathrm{V}$ (LcrV) antigens. $\mathrm{F} 1$ is the main protein component of the pseudocapsule, and is a dispensable virulence factor. The $V$ antigen is part of the Type Three Secretion System (TTSS), an essential virulence factor. Two subcutaneous doses of these F1/V vaccines provided $100 \%$ protection to mice against bubonic and pneumonic plague [15-17, 24]. However, when F1-V was tested in non-human primates, Cynomolgus macaques could be protected against aerosolized $Y$. pestis, but not African green monkeys [25]. Therefore, it is not yet clear whether $\mathrm{F} 1-\mathrm{V}$ could reliably provide protection in humans. F1-V and RYpVax have completed phases I and II trials, but the results of these trials are not yet available. RYpVax development has been halted, whereas $\mathrm{F} 1-\mathrm{V}$ marketing is announced for 2020. Another molecular vaccine has been patented by the Chicago University and is called V10. This vaccine, composed of a shorter form of the V antigen, provided protection against bubonic and pneumonic plague to mice and Cynomolgus macaques [26]. No phase I evaluation has yet been announced.

\section{Use of Yersinia pseudotuberculosis to vaccinate against Yersinia pestis}

\subsection{The vaccine strategy: a live $Y$. pseudotuberculosis administered orally}

The rationale for choosing this approach was to combine the immunogenicity and antigenic complexity of live vaccines with the genetic stability and possible oral administration of $Y$. pseudotuberculosis.

- A live vaccine

Live vaccines offer a series of advantages over recombinant vaccines. Preparedness plans against bioterrorist attacks imply stockpiling millions of vaccine doses. However, stockpiles have a finite lifespan and thus demand regular production of new doses, an expensive strategy [27]. Instead, live vaccines can be 
rapidly and easily produced in large amounts in an emergency situation, thus requiring more limited permanent stocks. For this reason, they are now viewed as a valuable alternative to molecular vaccines.

Subunit vaccines composed of a limited set of antigenic targets may not be effective in case of bacterial gene deletion and antigenic variation, as observed for the $\mathrm{F} 1$ and $\mathrm{V}$ antigens $[10,28]$. It is not the case for live vaccines, because their high antigenic complexity guarantees a response against a broad range of targets. In addition, live vaccines contain antigens in their native and naturally glycosylated molecular forms. Antigens are produced de novo as long as the bacteria persist, thus providing a prolonged stimulation of the immune system. Also, live vaccines do not require adjuvants since bacterial antigens (LPS and other pathogen-associated signatures) naturally stimulate the innate immune system. They induce both antibody and cell-mediated responses, and cooperation of these responses is more efficient for the elimination of pathogens [25].

Once developed and validated, live vaccines allow mass production at limited costs, a crucial condition for their use in endemic countries.

\section{- $\quad$ Y.pseudotuberculosis instead of $Y$.pestis}

One of the reasons that limit or prevent the use of $Y$. pestis as live vaccine is its genetic instability. Its genome is prone to frequent rearrangements and loss of genetic material, due to the presence of numerous insertion sequences [10, 12, 29], as described for the initial $Y$. pestis EV76 vaccine strain [30]. An alternative, first proposed by Jenner with his vaccinia vaccine against smallpox, was to use a microorganism different from the targeted pathogen (so it does not have its drawbacks), but closely related to it, to trigger cross-species protective immunity. Following this strategy, we decided to use $Y$. pseudotuberculosis, because its genome contains only few insertion sequences and is therefore much more stable than that of $Y$. pestis. Furthermore, $Y$. pseudotuberculosis [31] is much less virulent than the plague bacillus, and the oral route is its natural mode of transmission. Finally, the two species are genetically almost identical [31], with more than 95\% chromosomal identity [29], and therefore share a large variety of antigenic targets.

- A single dose vaccination

Most molecular vaccines require repeated injections and regular boosts to confer protection over long periods of time. Difficult to perform in the field, repeated injections are considered by public health authorities as a limitation for mass vaccination. Therefore, single dose vaccination represents a key advantage.

- An oral vaccination

Oral vaccination is also attractive because it avoids the use of syringes, which are a major source of disease transmission during mass vaccination campaigns, a problem frequently mentioned by WHO in its vaccination guidelines [32].

\subsection{Proof of concept: the naturally avirulent $Y$. pseudotuberculosis IP32680}

The IP32680 was first tested because it was a naturally attenuated strain showing no virulence in mice [33]. One oral IP32680 inoculation protected $75 \%$ of mice against bubonic plague. This validated the strategy that cross-protection provided by $Y$. pseudotuberculosis could protect against a fully virulent $Y$. pestis 
strain. However, two inoculations were required to induce high antibody titers and to protect $88 \%$ of mice against bubonic plague, and the IP32680 strain poorly protected against pneumonic plague. In addition, the causes of its attenuation were not known and the possibility of its reversion to full pathogenicity could not be excluded. These results prompted us to pursue in this direction and to develop an enhanced version of the vaccine.

\subsection{Attenuation of $Y$. pseudotuberculosis IP32953}

IP32953, the first sequenced Y.pseudotuberculosis strain [29], was used as a genetically defined strain to construct an irreversibly attenuated derivative. This was performed by allelic exchanges of virulence determinants with antibiotic resistance cassettes. The choice of virulence factors to delete was determined by the need to induce a strong loss of virulence, without losing important antigenic targets. Three virulence factors were chosen: the HPI, the YopK toxin, and the PsaA/pH6 antigen [34].

The HPI encodes the siderophore yersiniabactin that allow bacteria to acquire the necessary iron molecules from the iron-deprived environment of the host [35]. Loss of the HPI in the Y. pestis EV76 vaccine strain used by Girard and Robic in Madagascar was the cause of its attenuation [36]. The entire HPI was deleted using the homologous recombination technique [37].

The YopK toxin is part of the set of Yersinia outer membrane proteins (Yops) that are injected into the cell cytosol via the TTSS. Yops, which are encoded by the pYV/pCD1 virulence plasmid, target multiple intracellular signaling pathways, impairing both cytokines production and cell survival. YopK [38] is a regulator of Yop translocation and contributes to cell apoptosis [39]. YopK is also an inhibitor of inflammasome activation in targeted host cells [40], preventing the activation of caspase- 1 required to produce the IL-1B and IL-18 cytokines that are important for the host inflammatory response. As a consequence, absence of YopK facilitates the inflammatory and adaptive immune responses.

The pH6 antigen (PsaA) is a fibrillar antigen which, like $F 1$, homopolymerizes to form a fimbrial structure at the surface of the bacteria. In $Y$. pestis, it is part of the pseudocapsule and has adhesive properties to epithelial cells [41]. Its role in $Y$. pestis virulence depends on the route of infection [42-44].

Successive deletions of these virulence factors led to a gradual decrease of virulence of the V674 Y. pseudotuberculosis vaccine strain (Figure 1).

\subsection{Production of the $Y$. pestis F1 pseudocapsule by the $Y$. pseudotuberculosis vaccine strain}

The pseudocapsule produced by $Y$. pestis at $37^{\circ} \mathrm{C}$ is a polymer of the $\mathrm{F} 1$ subunit. $\mathrm{F} 1$ synthesis and assembly at the surface is encoded by the caf operon, which is located on the $Y$. pestis-specific pFra plasmid [9]. Although the $\mathrm{F} 1$ antigen confers anti-phagocytic properties to the bacteria in vitro [45], it has little or no impact on virulence [46-49]. Immunization with F1 confers protection against plague [50], and $Y$. pestis-infected mice can be treated with monoclonal antibodies against $F 1$ [51]. F1 is therefore an excellent target for protective immunity against $Y$. pestis.

F1-encapsulated $Y$. pseudotuberculosis strains were generated by introduction of the caf operon into V674. This was first obtained by inserting the caf locus into the 
pGEN expression plasmid, yielding the V674pF1 strain [34]. However, despite the plasmid was observed, resulting in a progressive reversion to un-encapsulated V674 bacilli. A sustained antibiotic pressure could be applied in vitro to ensure plasmid persistence, but this pressure could not be maintained in vivo. To overcome this difficulty, a VTnF1 version of V674 was generated by inserting the caf operon into the chromosome of V674 with a mini-transposon [52]. F1 was found to be homogeneously produced by VTnF1 individual colonies, in amounts comparable to those of $Y$. pestis [53].

\subsection{Virulence and persistence of the $\mathbf{V} \mathbf{6 7 4}$ vaccine strains}

While wild type Y. pseudotuberculosis strain IP32953 has a 50\% lethal dose $\left(L_{50}\right)$ for mice of $10^{8} \mathrm{CFU}$ by the oral route, the LD50s of its derivatives were systematically $>10^{10}$ CFU. Y. pseudotuberculosis colonizes the gut and Peyer's patches, and reaches the spleen and liver where it multiplies. All V674 derivatives were also able to colonize and persist for more than a week in the gut and the Peyer's patches $[33,34,53]$. They also retained the ability to breach the barriers of the Peyer's patches and to reach the spleen and liver $[33,53]$.

However, major differences in terms of histopathological lesions were seen between wild type and vaccine strains. IP32953 causes massive necrosis on day 6 in the Peyer's patches [34], with numerous abscesses and abundant inflammatory polymorphonuclear infiltration of the spleen and liver [33]. On the opposite, these organs were little affected by the attenuated strain V674.

Another key difference was that tissue bacterial loads of the V674 strains were at least $10^{3}$ lower than those of IP32953 (Figure 2 and [33]). After two weeks, none of the strains were detected in the spleen, indicating a robust response of the immune system that efficiently controlled bacteria. Y. pseudotuberculosis has a tropism for lymphatic tissues, including the spleen. The fact that attenuated strains penetrated but were subsequently eliminated from the spleen may explain their potent immunogenicity.

Whereas V674 and V674pF1 were still present in the feces and Peyer's patches of immunized mice after two weeks, VTnF1 almost completely disappeared from these sites, and no bacteria were detected in the feces during the six following months [53]. Because the only difference between $\mathrm{V} 674 \mathrm{pF} 1$ and $\mathrm{VTnF} 1$ is the stability of the encapsulation, the most likely reason for VTnF1 faster clearance is that the host eliminated encapsulated bacteria more easily.

\subsection{The immune response raised after oral administration of the Y. pseudotuberculosis vaccines}

The three genetically attenuated strains $\mathrm{V} 674, \mathrm{~V} 674 \mathrm{pF} 1$ and $\mathrm{VTnF} 1$ induced high and comparable IgG titers against antigens common to the three strains [34, 53]. The anti-F1 lgG response (for encapsulated strains) comes in addition to this "core" response. Actually, this anti-F1 antibody response is the major component of the humoral response to the vaccine because F1 is very immunogenic. Furthermore, the $\mathrm{F} 1$ antigen is shed and thus can be captured and processed by antigen-presenting cells distant from the site of infection. [34]. On the opposite, an F1 pseudocapsule surrounding bacterial cells may have masked other bacterial antigens. This was not the case since the $\lg \mathrm{G}$ response induced by $\mathrm{VtnF} 1$ was also directed against non-F1 
antigens. An immunoblot analysis of IgG target antigens revealed that least ten major produced $[33,53]$. This isotype profile indicates that the cellular immune response included (but was not limited to) Th1 lymphocytes [54], which produce the IFNy responsible for $\lg G 1$ to $\lg G 2$ commutation. $\lg A$ were also found in the serum and intestinal lavages [34], and probably contributed to the elimination of the vaccine from the intestine.

Sub-unit vaccines are better inducers of a humoral than a cellular response due to the use of Aluminum salts as adjuvant [55,56]. However, the cellular wing of the adaptive immune response plays an important role against plague $[57,58]$ and collaborates with the humoral defense to protect against pneumonic plague [59]. Live vaccines are good inducers of a cellular response. Memory cells induced by V674, V674pF1 and VTnF1 produced high levels of IFNy IL-17 and IL-10. IFNy is the central cytokine of the type 1 response (Th1), and is essential for both innate and adaptive immunity [60]. IL-17 attracts polymorphonuclear leukocytes and induces them to produce antimicrobial peptides. IL-17-producing $T$ lymphocytes (Th17) are essential to survive pneumonic plague [61,62]. On the opposite, IL-10 is the prototypical anti-inflammatory cytokine, which limits the adverse effects of inflammation induced by IFNy and IL-17 [63].

A clear recall response specific for the $\mathrm{F} 1$ antigen could be detected after vaccination with encapsulated strains $(\mathrm{V} 674 \mathrm{pF} 1$ \& VTnF1) [34, 53]. However, the cellular response against non-F1 antigens was much stronger, with IFNy and IL-17 levels similar to those induced by the non-specific $T$ cell stimulator Concanavalin $A$. Of note, the un-encapsulated V674 strain was already able to induce this memory, indicating that the presence of $\mathrm{F} 1$ was not essential for the recruitment of $Y$. pseudotuberculosis-specific $T$ lymphocytes. Despite the abundance of $F 1$, the strong response to other antigens may thus indicate that numerous antigens triggered their own specific cells, shaping a broad repertoire memory population.

\subsection{Protection against bubonic and pneumonic plague}

A one-dose vaccination with the naturally avirulent IP32680 strain or the genetically attenuated V674 protected mice against a subcutaneous (sc) infection, but did not confer full protection ( $<80 \%$; Figure 3 and [33, 34]). Because our goal was to achieve efficient protection against plague with a single-dose vaccination, we decided to add the highly immunogenic $Y$. pestis-specific F1 antigen to our vaccine strain.

V674pF1 and then VTnF1 used in a single intragastric (ig) dose $\left(10^{8} \mathrm{CFU}\right)$ provided $100 \%$ protection against pneumonic plague (challenge of $10^{5} Y$. pestis CFU, i.e. $30 \mathrm{LD}_{50}$ ). However, only $80 \%$ of the mice vaccinated with $\mathrm{V} 674 \mathrm{pF} 1$ survived a high-dose $\left(3,300 L_{50}\right)$ pneumonic plague challenge, whereas $100 \%$ of the animals vaccinated with VTnF1 did survive (Figure 3 and $[34,53]$. The difference of efficiency between $\mathrm{V} 674 \mathrm{pF} 1$ and $\mathrm{VTnF} 1$ was even more obvious against bubonic plague: only $80 \%$ of the mice vaccinated with $\mathrm{V} 674 \mathrm{pF} 1$ survived a moderate $\left(10^{3}\right.$ CFU; i.e. $100 \mathrm{LD}_{50}$ ) bubonic plague challenge, whereas, VTnF1 provided full protection. VTnF1 also protected $93 \%$ of the vaccinated mice against a high $(10,000$ $\mathrm{LD}_{50}$ ) bubonic plague challenge. The most likely explanation for this difference of protection is the more homogenous and sustained production of the F1 pseudocapsule by $\mathrm{VTnF1}$. The $\mathrm{F} 1$ antigen can activate macrophages [64], an 
adjuvant favorable for an adaptive immune response. Therefore, the efficiency of

Most subunit vaccines under test comprise the F1 antigen. Girard, who developed EV76, reported that the vaccine efficiency was directly related to the amount of F1 [36], possibly explaining why EV76 failed to protect against F1-negative Y. pestis [26, $48,65]$. An immune response focused on $F 1$ also favors the selection of F1-negative mutants [68].

We thus evaluated the immunity provided by VTnF1 against an F1-negative $Y$. pestis strain. We observed that vaccination conferred full protection to mice exposed to an F1-negative CO92, even at high-dose challenge of bubonic $(10,000$ $\left.\mathrm{LD}_{50}\right)$ and pneumonic plague $\left(3,000 \mathrm{LD}_{50}\right)$ [53]. Because VTnF1 is a whole-cell vaccine, this efficiency can be ascribed to its antigenic diversity. Antibodies recognizing multiple antigens other than $\mathrm{F} 1$ were evidenced [53]. VTnF1 also induced a strong cell-mediated immune response directed toward non-F1 antigens. Thus, protection against the F1-negative strain resulted from the cross-species immunity elicited by Y.pseudotuberculosis antigens shared with Y. pestis. Why attenuated $Y$. pestis induce an F1-focussed immune response, but not the attenuated F1-encapsulated $Y$. pseudotuberculosis VTnF1 remains an open question.

\subsection{Perspectives}

The next step is to remove the antibiotic-resistance cassettes that have been introduced into VTnF1 to delete important virulence genes and to insert the caf operon into the chromosome. Clinical-grade vaccine doses will then be produced on a large scale according to good manufacturing procedures to perform pre-clinical tests and ultimately start clinical tests.

\section{Conclusions}

Plague is a serious health problem for several countries in the world and a potential weapon of the bioterrorist threat. The VTnF1 live vaccine provides high-level 
protection against both bubonic and pneumonic plague after a single-dose immunization, and thus represents an efficient solution to prevent plague mortality. As VTnF1 is easy-to-produce, genetically stable, and irreversibly attenuated, it could be used both for vaccination campaigns in plague endemic countries, and as a fast response tool against a bioterrorist plague attack.

\section{Acknowledgements}

The authors thank Pierre Goossens and Xavier Montagutelli for helpful discussions. 


\section{References}

[1] J.M. Duplantier, J.B. Duchemin, S. Chanteau, E. Carniel, From the recent lessons of the Malagasy foci towards a global understanding of the factors involved in plague reemergence, Vet. Res., 36 (2005) 437-453.

[2] WHO, Human plague in 2002 and 2003, Wkly Epidemiol Rec, 79 (2004) 301-306.

[3] Anonymous, Select agents and toxins list. http://www.selectagents.gov/SelectAgentsandToxinsList.html, in: CDC (Ed.), Federal Select Agent Program, Washington, 2014.

[4] T.V. Inglesby, D.T. Dennis, D.A. Henderson, J.G. Bartlett, M.S. Ascher, E. Eitzen, A.D. Fine, A.M. Friedlander, J. Hauer, J.F. Koerner, M. Layton, J. McDade, M.T. Osterholm, T. O'Toole, G. Parker, T.M. Perl, P.K. Russell, M. Schoch-Spana, K. Tonat, Plague as a biological weapon - Medical and public health management, J. Am. Med. Assoc., 283 (2000) 2281-2290.

[5] R.E. Hoffman, J.E. Norton, Lessons learned from a full-scale bioterrorism exercise, Emerg Infect Dis, 6 (2000) 652-653.

[6] A. Guiyoule, G. Gerbaud, C. Buchrieser, M. Galimand, L. Rahalison, S. Chanteau, P. Courvalin, E. Carniel, Transferable plasmid-mediated resistance to streptomycin in a clinical isolate of Yersinia pestis, Emerg. Infect. Dis., 7 (2001) 43-48.

[7] M. Galimand, A. Guiyoule, G. Gerbaud, B. Rasoamanana, S. Chanteau, E. Carniel, P. Courvalin, Multidrug resistance in Yersinia pestis mediated by a transferable plasmid, N Engl J Med, 337 (1997) 677-680.

[8] T.J. Welch, W.F. Fricke, P.F. McDermott, D.G. White, M.L. Rosso, D.A. Rasko, M.K. Mammel, M. Eppinger, M.J. Rosovitz, D. Wagner, L. Rahalison, J.E. Leclerc, J.M. Hinshaw, L.E. Lindler, T.A. Cebula, E. Carniel, J. Ravel, Multiple antimicrobial resistance in plague: an emerging public health risk, PLoS ONE, 2 (2007) e309.

[9] K.F. Meyer, D.C. Cavanaugh, P.J. Bartelloni, J.D. Marshall, Jr., Plague immunization. I. Past and present trends, J Infect Dis, 129 (1974) Suppl:S13-18.

[10] A.P. Anisimov, L.E. Lindler, G.B. Pier, Intraspecific diversity of Yersinia pestis, Clin Microbiol Rev, 17 (2004) 434-464.

[11] J. Parkhill, B.W. Wren, N.R. Thomson, R.W. Titball, M.T.G. Holden, M.B. Prentice, M. Sebaihia, K.D. James, C. Churcher, K.L. Mungall, S. Baker, D. Basham, S.D. Bentley, K. Brooks, A.M. Cerdeno-Tarraga, T. Chillingworth, A. Cronin, R.M. Davies, P. Davis, G. Dougan, T. Feltwell, N. Hamlin, S. Holroyd, K. Jagels, A.V. Karlyshev, et al., Genome sequence of Yersinia pestis, the causative agent of plague, Nature, 413 (2001) 523-527.

[12] D. Zhou, Y. Han, E. Dai, Y. Song, D. Pei, J. Zhai, Z. Du, J. Wang, Z. Guo, R. Yang, Defining the genome content of live plague vaccines by use of whole-genome DNA microarray, Vaccine, 22 (2004) 3367-3374.

[13] V.A. Feodorova, L.V. Sayapina, M.J. Corbel, V.L. Motin, Russian vaccines against especially dangerous bacterial pathogens, Emerg Microbes Infect, 3 (2014) e86.

[14] P.C.F. Oyston, E.D. Williamson, S.E.C. Leary, S.M. Eley, K.F. Griffin, R.W. Titball, Immunization with live recombinant Salmonella typhimurium aroA producing F1 antigen protects against plague, Infect. Immun., 63 (1995) 563-568.

[15] M.J. Chiuchiolo, J.L. Boyer, A. Krause, S. Senina, N.R. Hackett, R.G. Crystal, Protective immunity against respiratory tract challenge with Yersinia pestis in mice immunized with an adenovirus-based vaccine vector expressing $V$ antigen, J. Infect. Dis., 194 (2006) 1249-1257. 
[16] J.E. Osorio, T.D. Powell, R.S. Frank, K. Moss, E.J. Haanes, S.R. Smith, T.E. Adamovicz, A.M. Friedlander, Protection Against Experimental Bubonic and Pneumonic Plague By a Recombinant Capsular F1-V Antigen Fusion Protein Vaccine, Vaccine, 16 (1998) 1131-1137.

[18] T. Une, R.R. Brubaker, Roles of $V$ antigen in promoting virulence and immunity in Yersiniae, J. Immunol., 133 (1984) 2226-2230.

[19] S.E.C. Leary, E.D. Williamson, K.F. Griffin, P. Russell, S.M. Eley, R.W. Titball, Active immunization with recombinant $\mathrm{V}$ antigen from Yersinia pestis protects mice against plague, Infect. Immun., 63 (1995) 2854-2858.

[20] H.S. Garmory, D. Freeman, K.A. Brown, R.W. Titball, Protection against plague afforded by immunisation with DNA vaccines optimised for expression of the Yersinia pestis V antigen, Vaccine, 22 (2004) 947-957.

[21] S.S. Bubeck, P.H. Dube, Yersinia pestis CO92 Delta yopH is a potent live, attenuated plague vaccine, Clin. Vaccine Immunol., 14 (2007) 1235-1238.

[22] Y. Flashner, E. Mamroud, A. Tidhar, R. Ber, M. Aftalion, D. Gur, S. Lazar, A. Zvi, T. Bino, N. Ariel, B. Velan, A. Shafferman, S. Cohen, Generation of Yersinia pestis attenuated strains by signature-tagged mutagenesis in search of novel vaccine candidates, Infect. Immun., 72 (2004) 908-915.

[23] S.W. Montminy, N. Khan, S. McGrath, M.J. Walkowicz, F. Sharp, J.E. Conlon, K. Fukase, S. Kusumoto, C. Sweet, K. Miyake, S. Akira, R.J. Cotter, J.D. Goguen, E. Lien, Virulence factors of Yersinia pestis are overcome by a strong lipopolysaccharide response, Nature Immunol., (2006).

[24] E.D. Williamson, S.M. Eley, K.F. Griffin, M. Green, P. Russell, S.E.C. Leary, P.C.F. Oyston, T. Easterbrook, K.M. Reddin, A. Robinson, R.W. Titball, A new improved sub-unit vaccine for plague: The basis of protection, FEMS Immunol. Med. Microbiol., 12 (1995) 223-230.

[25] S.T. Smiley, Current challenges in the development of vaccines for pneumonic plague, Expert Rev. Vaccines, 7 (2008) 209-221.

[26] C.A. Cornelius, L.E. Quenee, D. Elli, N.A. Ciletti, O. Schneewind, Yersinia pestis IS1541 transposition provides for escape from plague immunity, Infect. Immun., 77 (2009) 1807-1816.

[27] M.I. Metzer, The economics of Planning and Preparing for Bioterrorism, in: I.W. Fong, K. Alibek (Eds.) Bioterrorism and Infectious agents. A new dilemma for the 21st century. , Springer Sciences+Business Media, Inc., New-York, 2005, pp. 237257.

[28] C.C. Winter, W.B. Cherry, M.D. Moody, An Unusual Strain of Pasteurella pestis Isolated from a Fatal Human Case of Plague, Bull Org Mond Santé

Bull Wid Hlth Org, 23 (1960) 408-409.

[29] P.S. Chain, E. Carniel, F.W. Larimer, J. Lamerdin, P.O. Stoutland, W.M. Regala, A.M. Georgescu, L.M. Vergez, M.L. Land, V.L. Motin, R.R. Brubaker, J. Fowler, J. Hinnebusch, M. Marceau, C. Medigue, M. Simonet, V. Chenal-Francisque, B. Souza, D. Dacheux, J.M. Elliott, A. Derbise, L.J. Hauser, E. Garcia, Insights into the evolution of Yersinia pestis through whole-genome comparison with Yersinia pseudotuberculosis, Proc. Natl. Acad. Sci. U.S.A., 101 (2004) 13826-13831.

[30] Y. Cui, X. Yang, X. Xiao, A.P. Anisimov, D. Li, Y. Yan, D. Zhou, M. Rajerison, E. Carniel, M. Achtman, R. Yang, Y. Song, Genetic variations of live attenuated plague 
vaccine strains (Yersinia pestis EV76 lineage) during laboratory passages in different countries, Infect Genet Evol, 26 (2014) 172-179.

[31] M. Achtman, K. Zurth, C. Morelli, G. Torrea, A. Guiyoule, E. Carniel, Yersinia pestis, the cause of plague, is a recently emerged clone of Yersinia pseudotuberculosis, Proc. Natl. Acad. Sci. U.S.A., 96 (1999) 14043-14048.

[32] WHO, WHO-UNICEF-UNFPA joint statement on the use of auto-disable syringes in immunization services., (2003).

[33] T. Blisnick, P. Ave, M. Huerre, E. Carniel, C.E. Demeure, Oral vaccination against bubonic plague using a live avirulent Yersinia pseudotuberculosis strain, Infect Immun, 76 (2008) 3808-3816.

[34] A. Derbise, A. Cerdà Marín, P. Ave, T. Blisnick, M. Huerre, E. Carniel, C.E. Demeure, An encapsulated Yersinia pseudotuberculosis is a highly efficient vaccine against pneumonic plague, PLoS NTD, 6 (2012) e1528.

[35] E. Carniel, The Yersinia high-pathogenicity island: an iron-uptake island, Microbiol. Infect., 3 (2001) 561-569.

[36] G. Girard, L'immunité dans l'infection pesteuse. Acquisitions apportées par 30 années de travaux sur la souche de "Pasteurella pestis EV" (Girard et Robic), Biol. Méd., 52 (1963) 631-731.

[37] A. Derbise, B. Lesic, D. Dacheux, J.M. Ghigo, E. Carniel, A rapid and simple method for inactivating chromosomal genes in Yersinia, FEMS Immunol. Med. Microbiol., 38 (2003) 113-116.

[38] R.S. Dewoody, P.M. Merritt, M.M. Marketon, Regulation of the Yersinia type III secretion system: traffic control, Front Cell Infect Microbiol, 3 (2013) 4.

[39] K.N. Peters, M.O. Dhariwala, J.M. Hughes Hanks, C.R. Brown, D.M. Anderson, Early apoptosis of macrophages modulated by injection of Yersinia pestis YopK promotes progression of primary pneumonic plague, PLoS Pathog, 9 (2013) e1003324.

[40] I.E. Brodsky, N.W. Palm, S. Sadanand, M.B. Ryndak, F.S. Sutterwala, R.A. Flavell, J.B. Bliska, R. Medzhitov, A Yersinia Effector Protein Promotes Virulence by Preventing Inflammasome Recognition of the Type III Secretion System, Cell Host Microbe, 7 (2010) 376-387.

[41] F.Z. Liu, H.Q. Chen, E.M. Galvan, M.A. Lasaro, D.M. Schifferli, Effects of Psa and $\mathrm{F} 1$ on the adhesive and invasive interactions of Yersinia pestis with human respiratory tract epithelial cells, Infect. Immun., 74 (2006) 5636-5644.

[42] L.E. Lindler, M.S. Klempner, S.C. Straley, Yersinia pestis pH-6 Antigen Genetic, Biochemical, and Virulence Characterization of a Protein Involved in the Pathogenesis of Bubonic Plague, Infect. Immun., 58 (1990) 2569-2577.

[43] J.S. Cathelyn, S.D. Crosby, W.W. Lathem, W.E. Goldman, V.L. Miller, RovA, a global regulator of Yersinia pestis, specifically required for bubonic plague, Proc. Natl. Acad. Sci. U.S.A., 103 (2006) 13514-13519.

[44] A.P. Anisimov, I.V. Bakhteeva, E.A. Panfertsev, T.E. Svetoch, T.B. Kravchenko, M.E. Platonov, G.M. Titareva, T.I. Kombarova, S.A. Ivanov, A.V. Rakin, K.K. Amoako, S.V. Dentovskaya, The subcutaneous inoculation of $\mathrm{pH} 6$ antigen mutants of Yersinia pestis does not affect virulence and immune response in mice, J. Med. Microbiol., 58 (2009) 26-36.

[45] K.W. Carr, D.G. Heath, A.M. Friedlander, Antiphagocytic effect of F1 capsular protein of Yersinia pestis, Abstracts of the General Meeting of the American Society for Microbiology, 97 (1997) 96.

[46] A.M. Friedlander, S.L. Welkos, P.L. Worsham, G.P. Andrews, D.G. Heath, G.W. Anderson, M.L.M. Pitt, J. Estep, K. Davis, Relationship between virulence and 
immunity as revealed in recent studies of the $\mathrm{F} 1$ capsule of Yersinia pestis, Clin. Infect. Dis., 21 (1995) S178-S181.

[47] K.J. Davis, D.L. Fritz, M.L. Pitt, S.L. Welkos, P.L. Worsham, A.M. Friedlander, Pathology of experimental pneumonic plague produced by fraction 1-positive and fraction 1-negative Yersinia pestis in African green monkeys (Cercopithecus aethiops), Arch. Pathol. Lab. Med., 120 (1996) 156-163.

[48] L.E. Quenee, C.A. Cornelius, N.A. Ciletti, D. Elli, O. Schneewind, Yersinia pestis caf1 variants and the limits of plague vaccine protection, Infect. Immun., 76 (2008) 2025-2036.

[49] E.H. Weening, J.S. Cathelyn, G. Kaufman, M.B. Lawrenz, P. Price, W.E. Goldman, V.L. Miller, The Dependence of the Yersinia pestis Capsule on Pathogenesis Is Influenced by the Mouse Background, Infect. Immun., 79 (2011) 644-652.

[50] W.J. Simpson, R.E. Thomas, T.G. Schwan, Recombinant Capsular Antigen (Fraction-1) from Yersinia pestis Induces a Protective Antibody Response in BALB/C Mice, Am. J. Trop. Med. Hyg., 43 (1990) 389-396.

[51] J. Hill, C. Copse, S. Leary, A.J. Stagg, E.D. Williamson, R.W. Titball, Synergistic protection of mice against plague with monoclonal antibodies specific for the $\mathrm{F} 1$ and $V$ antigens of Yersinia pestis, Infect. Immun., 71 (2003) 2234-2238.

[52] K.H. Choi, J.B. Gaynor, K.G. White, C. Lopez, C.M. Bosio, R.R. KarkhoffSchweizer, H.P. Schweizer, A Tn7-based broad-range bacterial cloning and expression system, Nat Methods, 2 (2005) 443-448.

[53] A. Derbise, Y. Hanada, M. Khalife, E. Carniel, C.E. Demeure, Complete Protection against Pneumonic and Bubonic Plague after a Single Oral Vaccination, PLoS Negl Trop Dis, 9 (2015) e0004162.

[54] F.D. Finkelman, J. Holmes, I.M. Katona, J.F. Urban, Jr., M.P. Beckmann, L.S. Park, K.A. Schooley, R.L. Coffman, T.R. Mosmann, W.E. Paul, Lymphokine control of in vivo immunoglobulin isotype selection, Annu Rev Immunol, 8 (1990) 303-333.

[55] J.W. Mannhalter, H.O. Neychev, G.J. Zlabinger, R. Ahmad, M.M. Eibl, Modulation of the human immune response by the non-toxic and non-pyrogenic adjuvant aluminium hydroxide: effect on antigen uptake and antigen presentation, Clin Exp Immunol, 61 (1985) 143-151.

[56] G. Dinc, J.M. Pennington, E.S. Yolcu, M.B. Lawrenz, H. Shirwan, Improving the Th1 cellular efficacy of the lead Yersinia pestis $r F 1-V$ subunit vaccine using SA-41BBL as a novel adjuvant, Vaccine, 32 (2014) 5035-5040.

[57] S.J. Elvin, E.D. Williamson, Stat 4 but not Stat 6 mediated immune mechanisms are essential in protection against plague, Microb. Pathogen., 37 (2004) 177-184.

[58] Y. Levy, Y. Flashner, A. Tidhar, A. Zauberman, M. Aftalion, S. Lazar, D. Gur, A. Shafferman, E. Mamroud, T cells play an essential role in anti-F1 mediated rapid protection against bubonic plague, Vaccine, 29 (2011) 6866-6873.

[59] M.A. Parent, L.B. Wilhelm, L.W. Kummer, F.M. Szaba, I.K. Mullarky, S.T. Smiley, Gamma interferon, tumor necrosis factor alpha, and nitric oxide synthase 2, key elements of cellular immunity, perform critical protective functions during humoral defense against lethal pulmonary Yersinia pestis infection, Infect. Immun., 74 (2006) 3381-3386.

[60] S.M. Soudja, A.L. Ruiz, J.C. Marie, G. Lauvau, Inflammatory monocytes activate memory CD8(+) $\mathrm{T}$ and innate NK lymphocytes independent of cognate antigen during microbial pathogen invasion, Immunity, 37 (2012) 549-562. 
[61] J.S. Lin, L.W. Kummer, F.M. Szaba, S.T. Smiley, IL-17 contributes to cellmediated defense against pulmonary Yersinia pestis infection, J Immunol, 186 (2011) 1675-1684.

[62] D.R. Littman, A.Y. Rudensky, Th17 and regulatory T cells in mediating and restraining inflammation, Cell, 140 (2010) 845-858.

[63] M.J. McGeachy, K.S. Bak-Jensen, Y. Chen, C.M. Tato, W. Blumenschein, T. McClanahan, D.J. Cua, TGF-beta and IL-6 drive the production of IL-17 and IL-10 by T cells and restrain $\mathrm{T}(\mathrm{H})-17$ cell-mediated pathology, Nat Immunol, 8 (2007) 13901397.

[64] A. Sodhi, R.K. Sharma, H.V. Batra, U. Tuteja, Recombinant fraction 1 protein of Yersinia pestis activates murine peritoneal macrophages in vitro, Cell. Immunol., 229 (2004) 52-61.

[65] S.D. Knight, Structure and assembly of Yersinia pestis F1 antigen, Genus Yersinia: from Genomics to Function, 603 (2007) 74-87.

[66] S. McIntyre, S.D. Knight, F.L. J., Structure, assembly and applications of the polymeric F1 antigen of Yersinia pestis, in: E. Carniel, Hinnebusch B. J. (Ed.) Yersinia, molecular and Cellular Biology, Horizon Press, Wymondham, UK, 2004, pp. 363-407.

[67] T. Mangold, J. Goldberg, Plague wars. The Terrifying Reality of Biological Warfare. , St. Martin's Press, London, 2001.

[68] G.W. Anderson, P.L. Worsham, C.R. Bolt, G.P. Andrews, S.L. Welkos, A.M. Friedlander, J.P. Burans, Protection of mice from fatal bubonic and pneumonic plague by passive immunization with monoclonal antibodies against the F1 protein of Yersinia pestis, Am. J. Trop. Med. Hyg., 56 (1997) 471-473. 


\section{Legends to figures}

Figure 1: Contribution of individual gene deletion to IP32953 attenuation

The median lethal dose $\left(L_{50}\right)$ of the indicated strains by the oral route was measured by inoculating groups of mice with graded doses of bacteria and following survival for 21 days. The $\mathrm{LD}_{50}$ was calculated using the Spearman \& Karber method. The dotted line indicates the upper limit of the test.

Figure 2: Comparison of in vivo persistence for the successive vaccine strains Groups of mice were inoculated with a single oral dose of V674, V674pF1 or VTnF1 $\left(10^{8} \mathrm{CFU}\right)$ and were sacrificed at the indicated times to evaluate the bacterial loads in feces, Peyer's patches and spleen. Samples were minced and dilutions were plated on selective agar plates containing kanamycin to count colonies, with a detection limit of $10 \mathrm{CFU} / \mathrm{sample}$. Shown are individual values per pellet, per patch or in the whole spleen, respectively. The horizontal line indicates the median. The unpaired MannWhitney test was used for statistical analysis: Strains were compared by time and organ and only statistically significant comparisons are shown. ${ }^{*}: p \leq 0.05,{ }^{* *}: p<0.01$, ${ }^{* * *}: p<0.001,{ }^{* * *}: p<0.0001$.

Figure 3: Protective efficacy against bubonic and pneumonic plague achieved with the different $\mathrm{V} 674$ vaccine versions

Mice having received a single oral dose of $\mathrm{V} 674, \mathrm{~V} 674 \mathrm{pF} 1$ or $\mathrm{VTnF} 1\left(10^{8} \mathrm{CFU}\right)$ were challenged intranasally or subcutaneously to cause pneumonic or bubonic plague, respectively, with usual or high doses (severe challenge) of Y. pestis CO92, as indicated. Mouse survival was recorded daily for 21 days. The number of surviving mice/number of animals tested is indicated above the corresponding bar for each condition. The high dose challenge was only performed when $100 \%$ protection was obtained against the usual challenge. The Fisher Exact test was used for statistical analysis: *: $p \leq 0.05 ;{ }^{* *}: p<0.001$. 


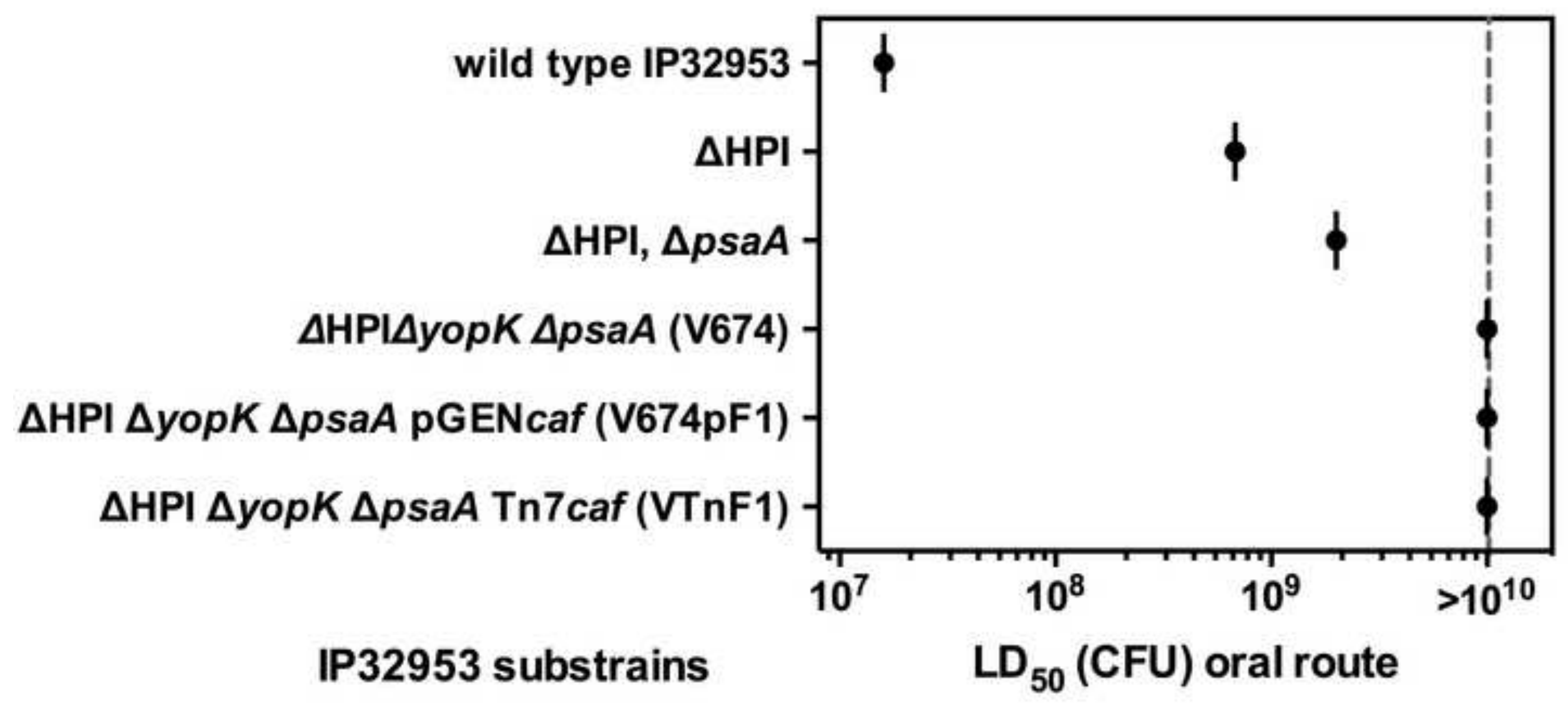


Feces

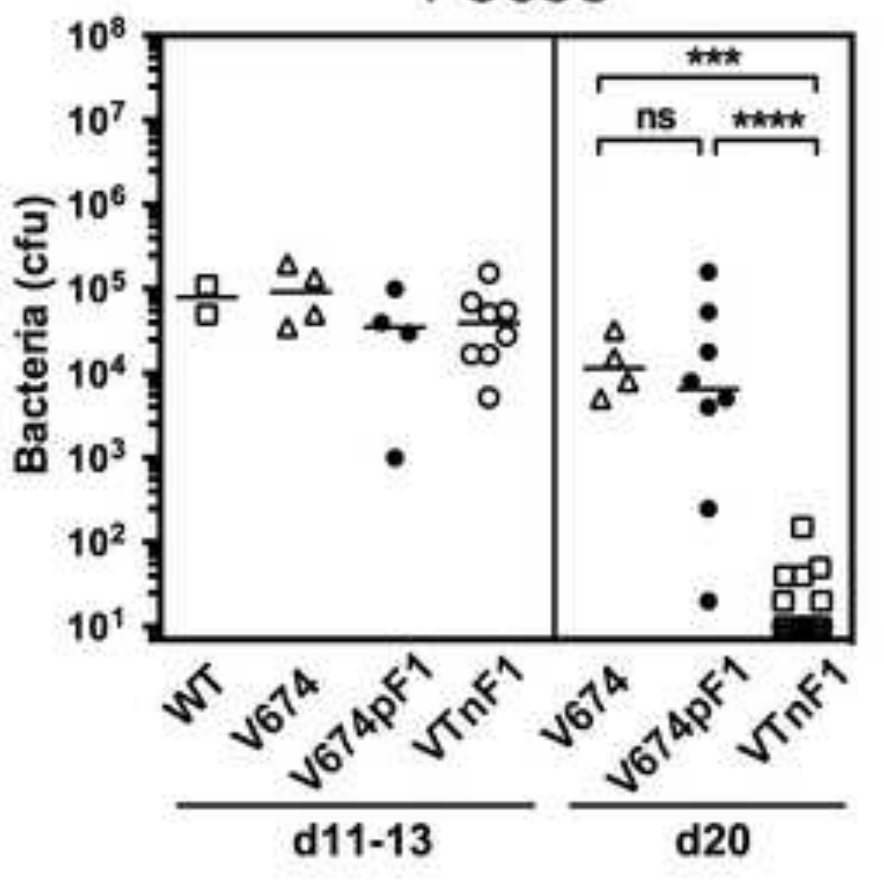

Peyer's patches

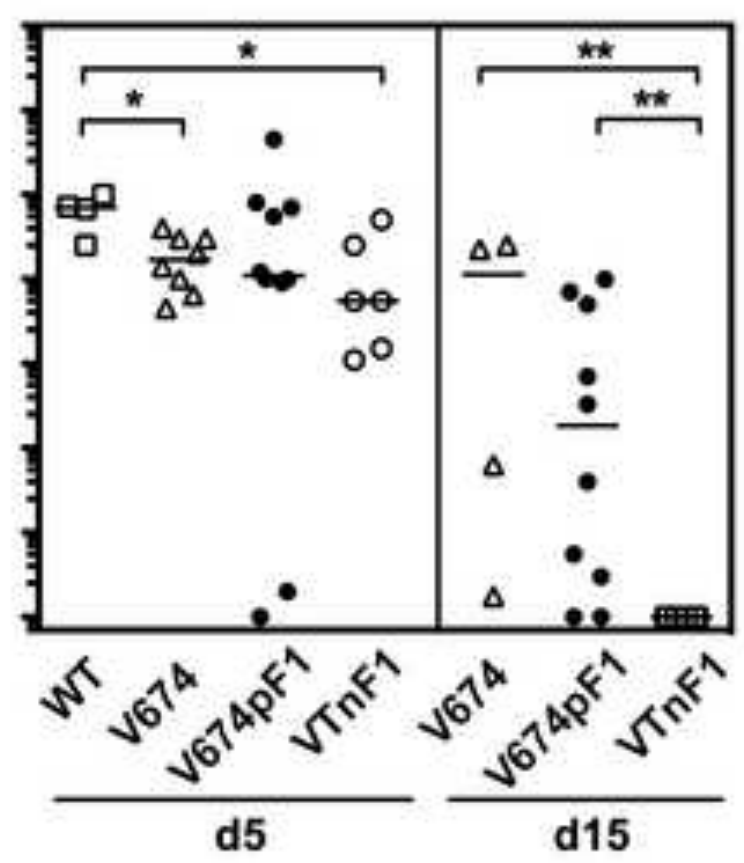

Spleen

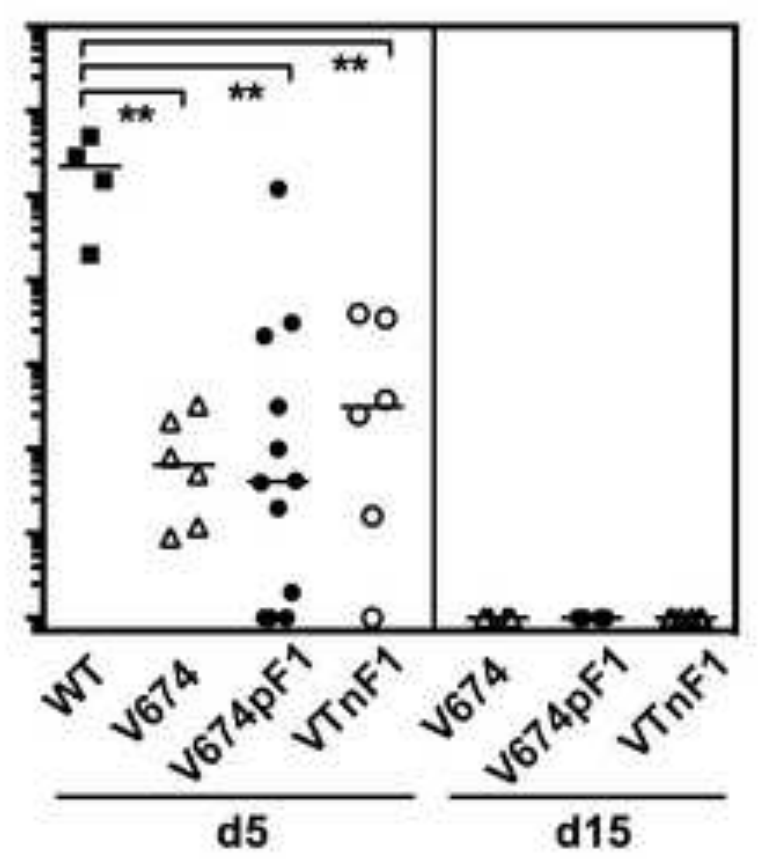


Bubonic plague

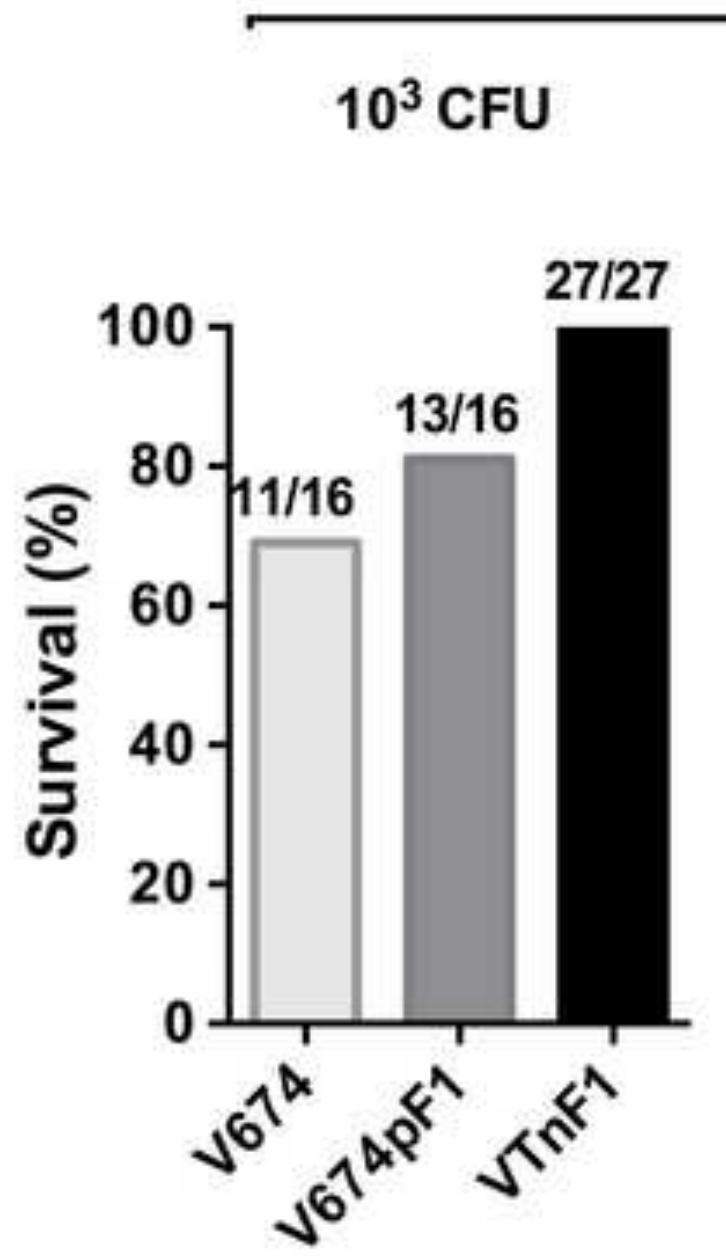

Pneumonic plague

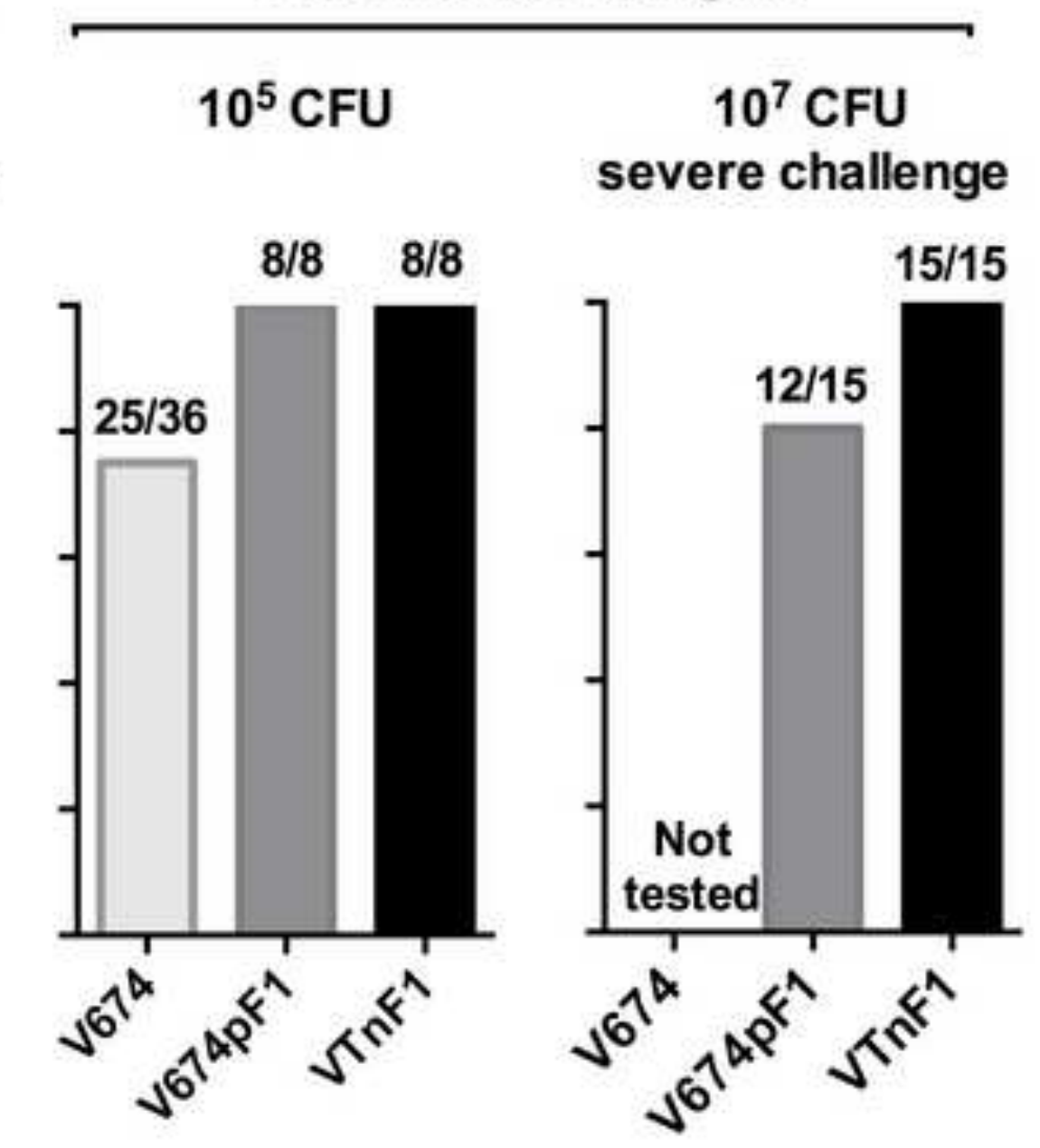

\section{$10^{5} \mathrm{CFU}$}

severe challenge

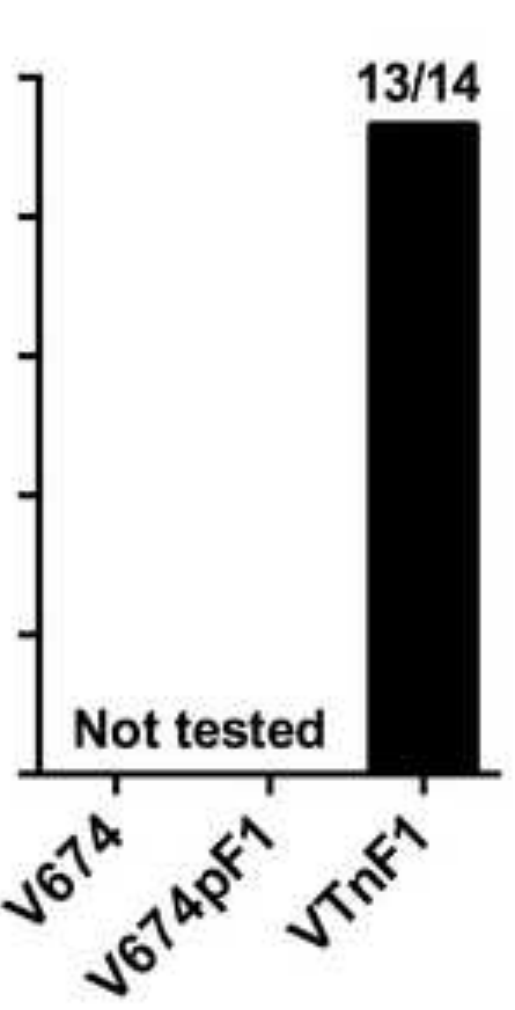

$13 / 14$ 